\title{
Relationship between articular eminence inclination and alterations of the mandibular condyle: a CBCT study
}

\section{Saione Cruz SA ${ }^{(a)}$ \\ Saulo Leonardo Sousa MELO(b) Daniela Pita de MELO(c) Deborah Queiroz FREITAS(a) Paulo Sérgio Flores CAMPOS(d)}

(a) Universidade de Campinas - Unicamp, Piracicaba Dental School, Department of Oral Diagnosis, Oral Radiology, Piracicaba, SP, Brazil.

(b) University of lowa College of Dentistry, Oral Pathology, Radiology and Medicine, lowa City, IA, USA.

(c) Universidade Estadual da Paraíba - UEPB, Department of Oral Diagnosis, Oral Radiology, Campina Grande, PB, Brazil.

(d) Universidade Federal da Bahia - UFBA, Department of Oral Diagnosis, Oral Radiology, Salvador, BA, Brazil.

Declaration of Interests: The authors certify that they have no commercial or associative interest that represents a conflict of interest in connection with the manuscript.

\section{Corresponding Author:}

Deborah Queiroz Freitas

E-mail: deborahq@unicamp.br

http://doi.org/10.1590/1807-3107BOR-2017.vol31.0025

Submitted: Sep 01, 2016

Accepted for publication: Feb 13, 2017

Last revision: Mar 03, 2017
Abstract: This study aimed at verifying the correlation among angulation of the articular eminence (AE), shape of the condyle and its degenerative bone diseases (DBDs), according to age and sex, through Cone Beam Computed Tomography (CBCT). Five hundred and twenty-eight temporomandibular joints (TMJ) were evaluated. The condyles were classified as: flat, convex, angled and rounded, and the AE angulation was measured. The DBDs evaluated were osteophytes, flattening, erosion, subcortical cysts and spinal sclerosis. There was no difference in the mean angulations in relation to age group $(\mathrm{p}>0.05)$. In age groups of $60-69$ years $(p=0.003)$ and 70 years or over $(p=0.021)$, the angulation was higher in males. There was an association between DBD and sex $(p=0.047)$, in that the prevalence was higher in females. Differences in AE angles were not observed in condyles with one or no DBDs $(\mathrm{p}>0.05)$. However, the presence of two or more DBDs led to a decrease in the angle $(\mathrm{p}<0.05)$. Angled condyles showed higher AE angulations than the flat and convex types $(p<0.01)$. In conclusion, the $\mathrm{AE}$ inclination is influenced by DBD and condyle shape; an association of two or more bone diseases in the condyle, or its flat or convex anatomy, results in a decrease in the angulation.

Keywords: Temporomandibular Joint; Mandibular Condyle; Cone-Beam Computed Tomography.

\section{Introduction}

In the opening and closing movements of the mouth, the condyle and the articular disc complex slide under the articular eminence (AE). The morphology of the AE enables this movement to flow naturally; it varies among individuals, and may change according to age, sex and masticatory function. ${ }^{1,2,3,4,5}$ The morphology of the bone components of the temporomandibular joint (TMJ) has been used to explain the origin of internal disorders and bone alterations according to the functional load in this area, as well as factors such as age and sex of the individual. $6,7,8$

The AE inclination constitutes an important element in the biomechanics of the TMJ, as well as the entire masticatory system, defined as the angle between the posterior wall of the $\mathrm{AE}$ and a horizontal reference plane. ${ }^{4,9}$ This inclination can be measured in degrees, with normal values between $30^{\circ}$ and $60^{\circ} \cdot{ }^{10}$ AEs below $30^{\circ}$ are classified as flat and above $60^{\circ}$, as steep ${ }^{9}$, 
and both classifications seem linked to internal articulation disorders. These disorders may lead to degenerative bone diseases (DBDs) in both the condyle and the AE. ${ }^{10}$

Articular bone diseases in the condyle may lead to adjustments in the AE inclination, as reported in some studies that have found joints with osteoarthritis to have more flat eminences. ${ }^{7}$ Therefore, condyles with changes such as erosions, flattening, osteophytes, subchondral cysts and bone sclerosis may be correlated with less steep eminences, as a result of the close relation between these two structures. ${ }^{11}$

Aside from being susceptible to DBDs, condyles may have varied morphology, which will influence the shape of the AE. Yale et al. ${ }^{12,13,14}$ developed a classification of condyle types, according to the wide variations in the shape of the top surface: flat, convex, angled, rounded. A previous study found that the $\mathrm{AE}$ inclination values are higher in joints without bone alterations than in joints with bone alterations. ${ }^{7}$ On the other hand, another study using the classification by Yale et $a{ }^{13}{ }^{13}$ found a greater mean value of $\mathrm{AE}$ inclination in males than in females, whereas no relation was found among factors of AE inclination, floor thickness of the mandibular fossa (FM) and the condyle morphology. ${ }^{8}$

Because of its three-dimensional complexity, the TMJ cannot be accurately evaluated by images that overlap. ${ }^{3,6}$ Currently, CBCT is the imaging modality of choice to investigate bone alterations of the TMJ, since it is possible to obtain sections of this structure on several levels. ${ }^{6,1516,1,7,18}$ Accordingly, the morphology of the osseous joint components, cortical bone integrity, and subcortical bone changes can be viewed using CBCT.

Further elucidation of the anatomy of structures, as well as the alterations of their functions and the relationship of these structures with external factors, is necessary to better address the morphological differences of TMJ bone components and the controversial relationship between AE inclination and condyle morphology, aspects that can be assessed more easily by today's modern diagnostic methods. To this end, the present study aimed mainly at analyzing the correlation among factors of AE angulation, condyle shape and the alterations in both. Additionally, we associated these data with age and sex, based on CBCT images.

\section{Methodology}

\section{Sample selection}

This was a retrospective cross-sectional study. It was previously approved by the local Ethics Committee on Human Research (Protocol \# 989172).

Two hundred and sixtyfour CBCT images of the maxillae of adult patients ages 20 to 82 years (mean age: 50.9; standard deviation: 12.68 ) were selected, resulting in $528 \mathrm{TMJs}$. The patients comprised 184 females (mean age: 50.8; standard deviation: 12.7 ) and 80 males (mean age: 51.2; standard deviation: 12.5). The images were selected by a single radiologist, in a private clinic, from exams acquired between 12/2012 and 03/2014. The following inclusion criteria was observed: TMJs had to be completely visible bilaterally; patients had to have all posterior teeth and could not present with any types of conditions, such as developmental disorders, fractures, anomalies, tumors or ankylosis in the region evaluated in the CBCT exams.

The CBCT exams were carried out on an i-CAT scanner with $120 \mathrm{kV}$ and 3-8 mA (Imaging Sciences International, Inc., Hatfield, USA), according to the protocol for the maxilla, $0.2 \mathrm{~mm}$ voxel, FOV (field of view) of $16 \times 6 \mathrm{~cm}$ and 40 seconds of acquisition time.

\section{Image evaluation}

The images were analyzed using Xoran ${ }^{\circledR}$ software (Xoran Technologies LLC, Ann Arbor, MI, USA) in a Eizo ${ }^{\circledR}$ S2100 (Eizo, Cypress, CA, USA), 21.3" monitor, at a resolution of $1280 \times 1024$ pixels.

The standard positioning of the images was performed by the researcher before the measurements were made by the examiners. The images were placed in a standard position in the multiplanar reconstruction (MPR), so that all the slices were made according to the correct positioning of the patient's head.

In the axial section, the patient's sagittal median plane (SMP) was aligned with the vertical reference line. In the sagittal section, the hard palate was positioned so that the researcher could see the anterior nasal spine (ANS) and the posterior nasal spine 
(PNS), and was then tilted to overlap the horizontal reference line. Accordingly, the palatine plane (PP) was at $0^{\circ}$ to the reference horizontal plane of the slice. Afterwards, the TMJ tool on the software was used to perform paracoronal and parasagittal slices ( $1 \mathrm{~mm}$ thick) of the condyle. The paracoronal slices were made along the long axis of the condyle, and the parasagittal slices were made perpendicular to the paracoronal plane (Figure 1).

Afterwards, two radiologists with over 5 years of experience in diagnosing $\mathrm{CBCT}$ evaluated the images individually. As part of their training, each examiner received verbal and written instructions on how to measure and evaluate each image. Some 50 images that were not part of the sample of 528 images were used at the time of the instructions to train each examiner's evaluation skills. After being instructed and calibrated, the examiners worked independently in a dark quiet environment.

Both examiners carried out three analyses of each TMJ: AE angulation, shape of condyle and presence of any DBDs, and recorded the information individually. Later, the examiners' responses were compared. In case of disagreement, the final diagnosis was made by consensus between them.

First analysis: Performed in a parasagittal slice from the center of the condyle ( $1 \mathrm{~mm}$ thick), using the TMJ tool to observe the articular eminence angle (AEA), formed by the internal angle between line 1 (connecting the articular eminence vertex and the mandibular fossa vertex) and line 2 (formed by PP',

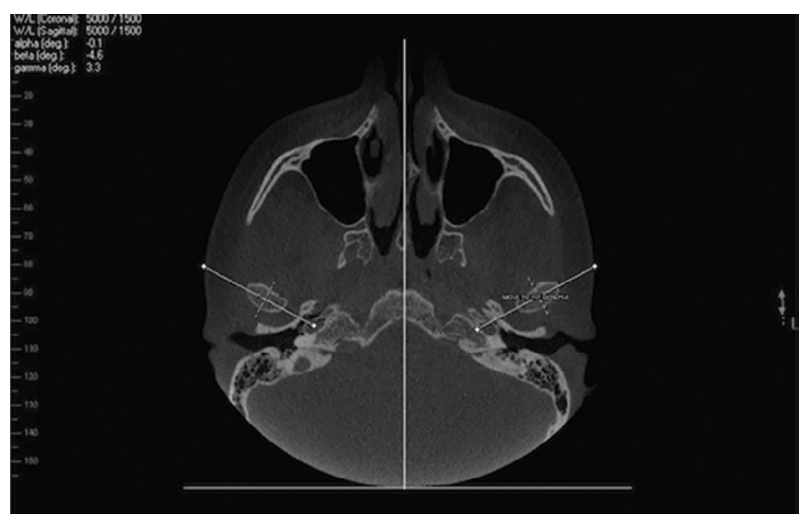

Figure 1. Paracoronal slice of the condyle (continuous line) and parasagittal slice of the condyle (dotted line). which is a line parallel to the palatine plane). The lines were obtained with Xoran ${ }^{\circledR}$ software tools (Figure 2).

Second analysis: To classify the condyle shape seen on the coronal slices, according to the Yale et al. (1966) classification (types A - flat, B - convex, C - angled, $D$ - rounded). The examiners used a template (Figure 3) developed with Inkscape software (Open Source Software licensed under the GPL., Vs. 0.91) to perform this evaluation. The shapes that did not fit any of the types (A, B, C or D) were classified as type E.

Third analysis: The DBDs were evaluated in parasagittal slices of condyle (1mm thick), using the TMJ tool to determine whether the condyles were normal (no change) or abnormal (when any of the following changes were detected: osteophytes, flattening, erosion, subcortical cysts and spinal sclerosis) (Figure 4).

\section{Statistical analysis}

The normality of data was initially checked using the Kolmogorov-Smirnov test to determine the ordinal variable (AEA, expressed as mean \pm standard error of the mean). Once the Gaussian distribution of the data was obtained, the comparison of mean values between the two groups was performed using Student's ttest. Comparison of the mean values among

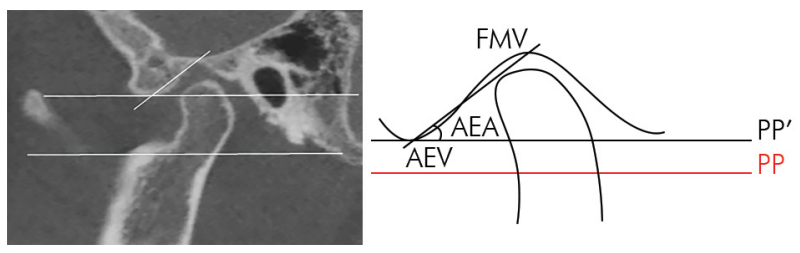

Figure 2. Measurement of the AEA formed between line 1 (formed by the connection points between the articular eminence vertex - AEV - and the mandibular fossa vertex - FMV) and line 2 (PP'- parallel to the palatine plane - PP).
A

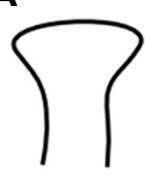

B

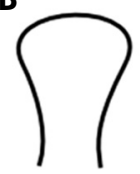

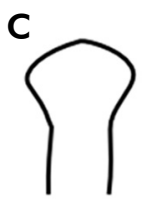

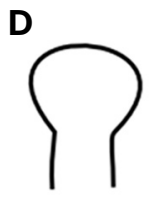

Figure 3. Layout/templates (simulating coronal slices) used by examiners to assess the type of condyle (A - flat, B - convex, C - angled, D - rounded). 
three or more groups was performed by analysis of variance (ANOVA test), followed by Tukey's multiple comparison test. The linear regression test was used to perform the analysis of correlation between the ordinal variables, applying the Pearson correlation coefficient. The chi-square test was used to analyze possible associations between the categorical variables (expressed as absolute and relative frequency). In all the cases, the differences were taken as significant when the $p$ value was less than 0.05 . Statistical analysis was performed using GraphPad Prism software, version 5.0.

\section{Results}

There was no significant difference in mean $\mathrm{AE}$ angulation according to the age of the individuals, either in the females $(p=0.136)$ or the males $(p=0.065)$, as seen in Figure 5.
However, the proximity of the $p$ value obtained for males to the threshold value (0.05), and the analysis of graphical data distribution, suggest a trend toward increased mean $\mathrm{AE}$ angulation with the aging of the individuals. The linear regression test was performed between the ages of the individuals and their $\mathrm{AE}$ angulation to confirm this trend. In fact, although the correlation observed (by a positive trend line) between the variables in the males $(r=0.156)$ was weak, it was considered significant $(p=0.048)$. On the other hand, the correlation between the variables in the females was too weak $(r=0.062)$ to be significant $(p=0.228)$.

Stratification of the mean angulations according to the different age groups to compare the $\mathrm{AE}$ angulation between the sexes, as shown in Table 1, indicated that only in older age groups (60 and over) was this measurement significantly higher in males than in females $(p<0.05)$.
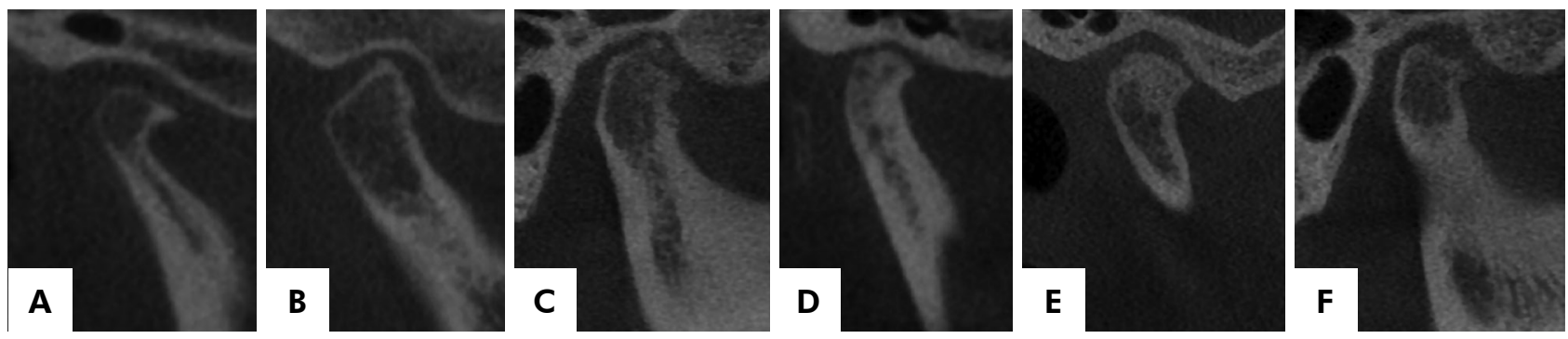

Figure 4. Degenerative bone diseases: A - osteophytes, B - flattening, C - erosion, D - subcortical cysts, E - spinal sclerosis, $\mathrm{F}$ - combination of two pathological changes (osteophytes and erosion).

A

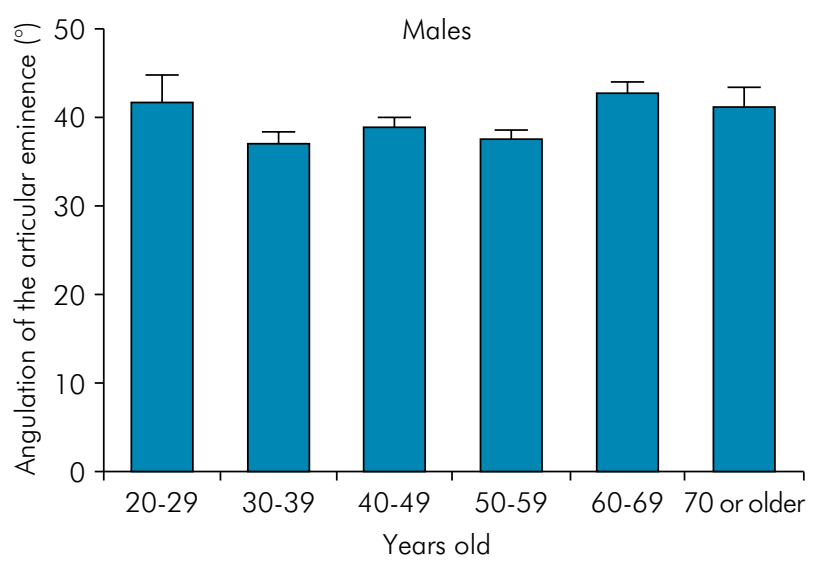

B

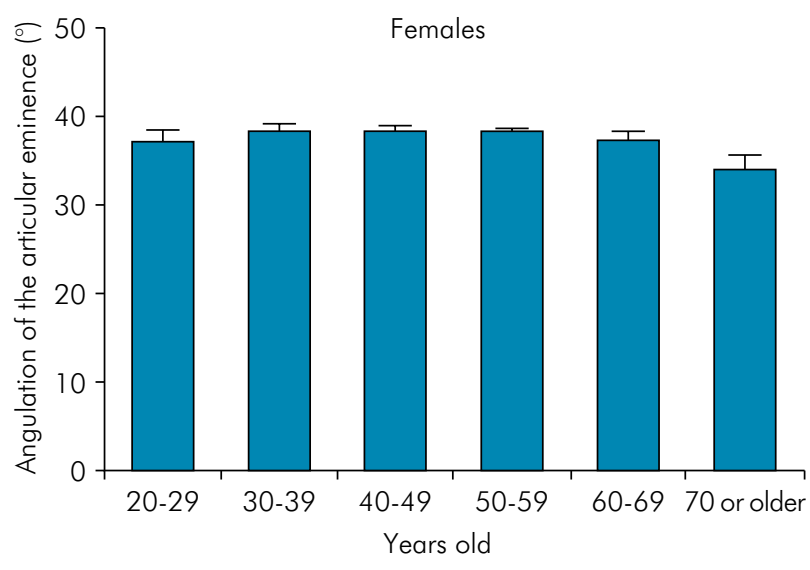

Figure 5. Mean AE angulation of males and females, according to the age groups. One-way ANOVA indicated no difference between the age decades ( $p=0.065$ and 0.136 , respectively). 
When comparing the sides, there was no significant difference in $\mathrm{AE}$ angulation degree between the right and left sides according to Student's $t$ test $(p=0.213)$. It was observed that $47.7 \%$ of the condyles studied had DBDs. There was no significant association $(p=0.663)$ between frequency of these changes and the affected side (Table 2), but there was a significant association $(p=0.0472)$ between these changes and the sex of the subjects (Table 3).

Flattening was the most frequent change observed in the condyles (153 cases, corresponding to $60.7 \%$ ), followed by osteophytes (122 cases, representing $48.4 \%$ ) and subcortical cysts (33 cases, representing 13.1\%). There were only 19 cases of erosion $(7.5 \%), 6$ cases of sclerosis $(2.3 \%)$ and 2 of free bodies $(0.8 \%)$. However, it is noteworthy that combinations of two or more DBDs were observed in 93 cases (17.6\%).

No significant differences were observed between the means of $\mathrm{AE}$ angulations and condyles either with DBD or without the alterations under study $(p>0.05)$, as shown in Figure 6. However, the combination of two or more pathological changes in the condyle was especially interesting, since it caused a reduction in mean AE angulations $(p<0.01)$ which did not differ from the isolated $\mathrm{DBD}$, regardless of type.

Considering the morphology of the $\mathrm{AE}$ in the individuals assessed, it was observed that 58.5\% of the cases (309) were type A, 9.5\% (50 cases) type B, $8.7 \%$ (46 cases) type C, $18.0 \%$ (95 cases) type D, and $5.3 \%$ (28 cases) type E. When comparing the means of $\mathrm{AE}$ angulations in relation to these different condyle morphological patterns (Figure 7), it was observed that the mean of type C condyles (angled) was significantly greater than type A (flat) and B condyles (convex) $(\mathrm{p}<0.01)$. The comparison between the other types revealed no significant differences in mean $\mathrm{AE}$ angulations ( $\mathrm{p}>0.05)$.

Table 1. Mean ( \pm standard error) of $A E$ angulation according to sex and age.

\begin{tabular}{lccccccc}
\hline \multirow{2}{*}{ Sex } & \multicolumn{7}{c}{ Age Group } \\
\cline { 2 - 6 } & $20-29$ & $30-39$ & $40-49$ & $50-59$ & $60-69$ & $70+$ & General mean \\
\hline Female & $37.1 \pm 1.1$ & $38.3 \pm 0.9$ & $38.2 \pm 0.9$ & $38.0 \pm 0.6$ & $37.3 \pm 1.0$ & $33.8 \pm 1.8$ & $37.6 \pm 0.4$ \\
Male & $41.6 \pm 3.2$ & 37.01 .3 & $39.0 \pm 1.0$ & $37.7 \pm 0.3$ & $42.6 \pm 1.3$ & $41.3 \pm 2.1$ & $39.3 \pm 0.5$ \\
p-value* & 0.134 & 0.422 & 0.607 & 0.797 & 0.003 & 0.021 & 0.016 \\
\hline
\end{tabular}

*According to student's $t$ test.

Table 2. Distribution of degenerative bone diseases in the affected side of the condyles.

\begin{tabular}{lccc}
\hline \multirow{2}{*}{ Affected side } & No alterations & With alterations & \multirow{2}{*}{ Total } \\
\cline { 2 - 3 } & $\mathrm{n}(\%)$ & $\mathrm{n}(\%)$ & \\
\hline Right side & $135(25.6 \%)$ & $129(24.4 \%)$ & 264 \\
Left side & $141(26.7 \%)$ & $123(23.3 \%)$ & 264 \\
Total & $276(52.3 \%)$ & $252(47.7 \%)$ & 528 \\
p-valor (qui-square) & & 0.663 & \\
\hline
\end{tabular}

Table 3. Distribution of degenerative bone diseases in condyles related to sex.

\begin{tabular}{lccc}
\hline \multirow{2}{*}{ Sex } & No alterations & With alterations & Total \\
\cline { 2 - 4 } & $\mathrm{n}(\%)$ & $\mathrm{n}(\%)$ & $\mathrm{n}(\%)$ \\
\hline Male & $94(58.75 \%)$ & $66(41.25 \%)$ & $160(100 \%)$ \\
Female & $180(48.9 \%)$ & $188(51.1 \%)$ & $368(100 \%)$ \\
Total & $276(52.3 \%)$ & $252(47.7 \%)$ & \\
p-valor (qui-square) & & 0.0472 & \\
\hline
\end{tabular}

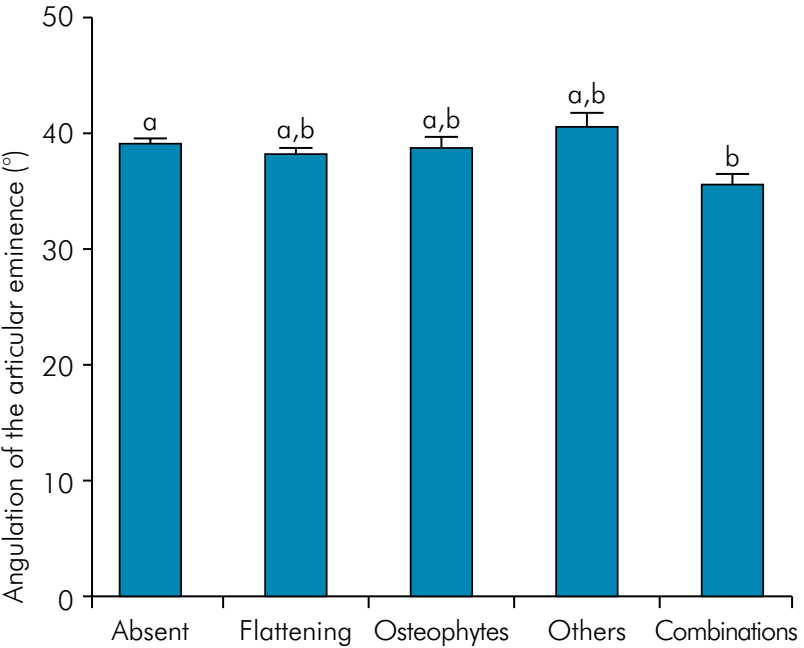

Figure 6. Distribution of $\mathrm{AE}$ angulation (mean \pm standard error) of the subjects analyzed, according to types of DBD present in the condyles. Different letters represent statistical differences (ANOVA, $\mathrm{p}=0.006$; and Tukey's multiple comparison test). 


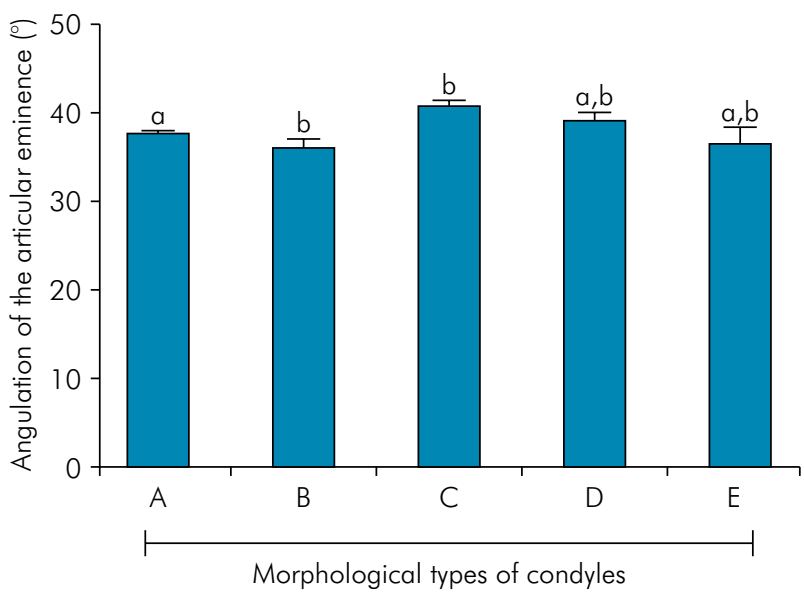

Figure 7. Distribution of $A E$ angulations (mean \pm standard error) related to the morphological type of condyle. Type A - flat; type B - convex; type C - angled; type D - rounded; and type E: other shapes. Different letters represent significantly different mean values (ANOVA, $p=0.0007$; and Tukey's multiple comparison test).

\section{Discussion}

The CBCT is currently considered the imaging modality of choice for visualization of TMJ bone structures. However, there are a great variety of protocols that may be selected for image acquisition, varying mainly in relation to field of view and voxel size. Considering that voxel sizes $0.076 \mathrm{~mm}$ and $0.3 \mathrm{~mm}$ showed no significant differences in the accuracy of the diagnosis of bone changes in TMJs, ${ }^{15}$ we used images obtained with $0.2 \mathrm{~mm}$ voxel, which allowed the observation of bone changes. Regarding the field of view, the use of a relative wide area is justified because both left and rights sides of the TMJ have to be displayed. ${ }^{6,8}$ Two main methods are described in the literature to measure the $\mathrm{AE}$ inclination: the "top-roof line method," obtained by connecting the lowest point of the $\mathrm{AE}$ and the highest point of the FM; and the "best-fit line method," obtained by adjusting a line drawn to the posterior slope of the AE. The angle is measured between the selected line and a horizontal reference plane. Importantly, both methods have shown similar AE angle values. ${ }^{7,8,19}$

The inclination of the AE is therefore defined as the angle formed by one of the lines that passes through the AE and the Frankfort horizontal plane or any other horizontal plane, such as the occlusal plane or palatal plane $^{9}$. The horizontal plane used as the reference in this study was the palatal plane (formed between the anterior nasal spine and posterior nasal spine), since it is easy to define in multiplanar reconstruction in the axial and sagittal slices of the software used. In contrast, Estomaguio et al., ${ }^{2}$ Csadó et al., ${ }^{4}$ Çağlayan et al. ${ }^{7}$ and Sümbüllü et al..$^{19}$ used the Frankfort horizontal plane (formed by a line connecting the porion to the infraorbital foramen) as the reference plane. In our study, the Frankfort horizontal plane was not used, because the infraorbital foramen was out of the field of view in most of the exams studied.

Comparing the right and left sides of the TMJ, there were no differences in the mean angulation of the AEs, which is similar to that found by Csadó et al. ${ }^{4}$ In addition, the comparison between the right and left sides in females and males also showed no statistical differences between the means of the angles, as corroborated by Wu et al. ${ }^{3}$

A tendency for the $\mathrm{AE}$ angle to increase above the age of 30 years was observed in males. Our results agree with those reported by Wu et al., ${ }^{3}$ who found a moderate correlation between age and $\mathrm{AE}$ angulation in males, and also observed a positive correlation in women. Conversely, there was no positive correlation in our study between female angles and age, which can be explained by the differences in the populations studied: Wu et al. ${ }^{3}$ evaluated 95 exams of Asian female patients (aged 11 and 88 years), whereas we analyzed 184 exams of Brazilian female patients (aged 20 to 82 years). Hence, we examined a larger sample of different ethnicity and did not include ages younger than 20 years, since the joints are not fully formed before then.

Males have significantly higher angle values than women only in older age groups, corroborating in part the study by Ilguy et al. ${ }^{8}$, who asserted that the mean $\mathrm{AE}$ angulation in males is significantly higher than in females; however, their means were not separated by age groups. On the other hand, the results found by Csadó et al. ${ }^{4}$ showed no significant difference in angulation values between the sexes. However, it is noteworthy that these authors included a group of edentulous patients in their sample, and used the panoramic radiograph to analyze the angles. The differences between the number of subjects in the female and the male groups should not be a concern, because the 
statistical analysis already considers and weighs these differences to evaluate a possible association with factors such as sex. Therefore, the results are reliable and the difference in the distribution of individuals in both groups had no effect on the results.

Alterations in the condyles did not affect the AE angulations. However, when two or more bone changes were present in the condyles, an average reduction in AE angulation was observed, probably because two or more bone changes cause more severe alterations. Estomaguio et al. ${ }^{2}$ reported a similar correlation when they studied 59 patients, 39 with and 20 without bone changes, stating that $\mathrm{AE}$ angulation in patients with bone change was significantly lower than in patients without bone change. However, the authors did not specify if there was more than one bone alteration in the condyle. Similarly, Çağlayan et al. ${ }^{7}$ reported that the value of $\mathrm{AE}$ angulation in TMJs without dysfunction was higher than in TMJs with dysfunction, but no statistically significant difference was verified.

In regard to the bone changes in the condyle, females have been found to have more. In a sample similar to that of the present study (randomized subjects at a time interval), Estomaguio et al. ${ }^{2}$ also observed more women with (66.1\%) than without (33.8\%) DBD.

Regarding the morphology of the condyle according to the classification by Yale et al., ${ }^{13}$ the present study found that most of the condyles presented flat morphology, in contrast to Yale et al., ${ }^{13}$ who observed angled morphology to be more prevalent $(43 \%)$ in pre-Hispanic populations, and convex types, more prevalent in Caucasians (50.2\%) and blacks (72\%), in their sample of European patients. It is noteworthy that $28.9 \%$ of the condyles in our sample presented flattening, namely that of the upper portion, which may have resulted in the higher prevalence of flattened shapes in the sample.

\section{References}

1. Pandis N, Karpac J, Trevino R, Williams B. A radiographic study of condyle position at various depths of cut in dry skulls with axially corrected lateral tomograms. Am J Orthod Dentofacial Orthop. 1991;100(2):116-22. https://doi.org/10.1016/S0889-5406(05)81518-5
Observing the morphological patterns of the condyles and their relationship to mean AE angulation, the differences were between angled condyles, which presented higher mean angulations than flat and convex condyles. When the authors analyzed the anatomic shape of each type of condyle, it was expected that the angled form would be related to higher angulation, since this type is apparently more voluminous than the others, especially more than the flat and convex types, as verified by imaging analysis. However, a previous study found no correlation between condyle morphology and $\mathrm{AE}$ angulation. ${ }^{8}$ It is noteworthy that important methodological differences between the studies, such as number of evaluators and sample size, may have contributed to such divergent results.

The main clinical contributions offered by this study to the professionals of the field regard the relationship between the $\mathrm{AE}$ angulation and the condyle, given its importance in TMJ biomechanics. They should be well-enough informed to know that this inclination is influenced by DBD and by the condyle shape, considering that an association of two or more bone diseases in the condyle, or its flat or convex anatomy, decreases the angulation.

\section{Conclusion}

The presence of two or more bone diseases in the condyle results in a reduction in $\mathrm{AE}$ angulation. Moreover, the shape of the condyle influences the $\mathrm{AE}$ inclination, seeing that the angled condyle is associated with higher $\mathrm{AE}$ angulation than the flat and convex types. Additionally, the AE angulation is not influenced by age, but is influenced by the sex, seeing that it is higher in males over 60 years old. Concerning DBDs, their propensity is greater among females. 
3. Wu CK, Hsu JT, Shen YW, Chen JH, Shen WC, Fuh LJ. Assessments of inclinations of the mandibular fossa by computed tomography in an Asian population. Clin Oral Investig. 2012;16(2):443-50. https://doi.org/10.1007/s00784-011-0518-y

4. Csadó K, Márton K, Kivovics P. Anatomical changes in the structure of the temporomandibular joint caused by complete edentulousness. Gerodontology. 2012;29(2):111-6. https://doi.org/10.1111/j.1741-2358.2011.00498.x

5. Kranjěić J, Vojvodić D, Žabarović D, Vodanović M, Komar D, Mehulić K. Differences in articular-eminence inclination between medieval and contemporary human populations. Arch Oral Biol. 2012;57(8):1147-52. https://doi.org/10.1016/j.archoralbio.2012.05.009

6. Ejima K, Schulze D, Stippig A, Matsumoto K, Rottke D, Honda K. Relationship between the thickness of the roof of glenoid fossa, condyle morphology and remaining teeth in asymptomatic European patients based on cone beam CT data sets. Dentomaxillofac Radiol. 2013;42(3):90929410. https://doi.org/10.1259/dmfr/90929410

7. Çağlayan F, Sümbüllü MA, Akgül HM. Associations between the articular eminence inclination and condylar bone changes, condylar movements, and condyle and fossa shapes. Oral Radiol. 2014;30(1):84-91. https://doi.org/10.1007/s11282-013-0149-x

8. İlgüy D, İlgüy M, Fişekçioğlu E, Dölekoğlu S, Ersan N. Articular eminence inclination, height, and condyle morphology on cone beam computed tomography. Sci World J. 2014;2014:761714. https://doi.org/10.1155/2014/761714

9. Katsavrias EG. Changes in articular eminence inclination during the craniofacial growth period. Angle Orthod. 2002;72(3):258-64. https://doi.org/10.1043/00033219(2002)072<0258:CIAEID>2.0.CO;2

10. Ozkan A, Altug HA, Sencimen M, Senel B. Evaluation of articular eminence morphology and inclination in TMJ internal derangement patients with MRI. Int J Morphol. 2012;30(2):740-4. https://doi.org/10.4067/S0717-95022012000200064

11. Boer EW, Dijkstra PU, Stegenga B, Bont LG, Spijkervet FK. Value of cone-beam computed tomography in the process of diagnosis and management of disorders of the temporomandibular joint. Br J Oral Maxillofac Surg. 2014;52(3):241-6. https://doi.org/10.1016/j.bjoms.2013.12.007

12. Yale SH, Rosenberg HM, Ceballos M, Haupt-Fuehrer JD. Laminagraphic cephalometry in the analysis of mandibular condyle morphology: a preliminary report. Oral Surg Oral Med Oral Pathol. 1961;14(7):793-805. https://doi.org/10.1016/S0030-4220(61)80008-X

13. Yale SH, Ceballos M, Kresnoff CS, Hauptfuehrer JD. Some observations on the classification of mandibular condyle types. Oral Surg Oral Med Oral Pathol. 1963;16(5):572-7. https://doi.org/10.1016/0030-4220(63)90146-4

14. Yale SH, Allison BD, Hauptfuehrer JD. An epidemiological assessment of mandibular condyle morphology. Oral Surg Oral Med Oral Pathol. 1966;21(2):169-77. https://doi.org/10.1016/0030-4220(66)90238-6

15. Lukat TD, Perschbacher SE, Pharoah MJ, Lam EW. The effects of voxel size on cone beam computed tomography images of the temporomandibular joints. Oral Surg Oral Med Oral Pathol Oral Radiol. 2015;119(2):229-37. https://doi.org/10.1016/j.oooo.2014.10.015

16. Honda K, Larheim TA, Maruhashi K, Matsumoto K, Iwai K. Osseous abnormalities of the mandibular condyle: diagnostic reliability of cone beam computed tomography compared with helical computed tomography based on an autopsy material. Dentomaxillofac Radiol. 2006;35(3):152-7. https://doi.org/10.1259/dmfr/15831361

17. Cömert KiliçS, Kiliç N, Sümbüllü MA. Temporomandibular joint osteoarthritis: cone beam computed tomography findings, clinical features, and correlations. Int J Oral Maxillofac Surg. 2015;44(10):1268-74. https://doi.org/10.1016/j.ijom.2015.06.023

18. Merigue LF, Conti AC, Oltramari-Navarro PV, Navarro RL, Almeida MR. Tomographic evaluation of the temporomandibular joint in malocclusion subjects: condylar morphology and position. Braz Oral Res. 2016;30(1):S1806-83242016000100222. https://doi.org/10.1590/1807-3107BOR-2016.vol30.0017

19. Sümbüllü MA, Cağlayan F, Akgül HM, Yilmaz AB. Radiological examination of the articular eminence morphology using cone beam CT. Dentomaxillofac Radiol. 2012;41(3):234-40. https://doi.org/10.1259/dmfr/24780643 